\title{
ON THE IDENTIFICATION OF CONTINUOUS VIBRATING SYSTEMS MODELLED BY HYPERBOLIC PARTIAL DIFFERENTIAL EQUATIONS*
}

BY

F. E. UDWADIA (University of Southern California)

AND

D. K. SHARMA ( Bell Laboratories, Murray Hill, New Jersey)

1. Introduction. The identification of spatially varying parameters in systems that can be described by hyperbolic equations has attracted a considerable amount of interest in recent years [1-5]. The main interest in this area is to make improved predictions of the response of continuous mechanical and structural systems subjected to various types of dynamic loads. Such predictions naturally require an adequate knowledge of the parameters which are involved in the system model.

The problem of parametric identification then consists of estimating the spatially varying parameters. This estimation is generally done by minimizing the difference (often suitably weighted) between the time history of the response of the physical system (measured at one or more points, to one or more test inputs) and that of the mathematical model. The form of the governing equations that describe the system behavior are assumed to be known a priori.

Such a minimization problem can be construed in the framework of optimization theory [6], and the complete arsenal of nonlinear programming techniques can be brought to bear on solving the problem in a computationally efficient manner [2]. However, minimizing the mismatch in the time history of the response (at one or more points) between the system and the model does not necessarily guarantee that the mathematical model coincide with that of the real system [3]. This then leads to the problem of establishing the conditions regarding the nature of the measurements and the location(s) at which they should be made, such that the spatially varying parameters can be uniquely identified from the response data.

This paper treats systems of finite spatial extent which can be modelled by the one dimensional, second order wave equation. Such equations arise commonly in various areas of mathematical physics such as acoustics, elastic wave propagation, and electromagnetic theory. Two different questions have been addressed. The first deals with 'partial

\footnotetext{
* Received June 21, 1983.
} 
identification' and inquires into the possibility of retrieving all the eigen-values of the system from time response data. The second deals with the identification of the distributed coefficients. Sufficient conditions for the unique determination of all the eigenvalues of the system are obtained, and conditions are determined under which the coefficients can be uniquely identified by suitable measurements made at one point in the spatial domain.

Studies done to date [e.g., 7-9] in this area have primarily concentrated on using response data obtained from impulsive (delta-function) loads applied at the "free" end of the system. Recognizing the difficulty often encountered in the creation of such a load, we consider a wider class of loading conditions. Also, we consider a two-point boundary value problem representing a finite spatial domain rather than a semi-infinite domain. Furthermore, the partial identification theorem when applied to two different sets of boundary conditions guarantees complete identification of the system [19].

A sizeable body of literature exists on the identification of distributed systems of the parabolic type [20] ${ }^{1}$. Kitamura and Nakagiri [21] have studied the two-point boundary value problem using, largely, set theoretic methods to establish conditions under which unique identification of some of the coefficients is possible. While their approach fundamentally differs from ours, the work of Pierce [22] on parabolic differential equations, in many ways, parallels the development in this paper. However, the results presented by us, besides being applicable to a different type of differential equation, are more extensive and bear greater generality. Kobayashi [23] tackles a slightly different identification task wherein he tries to represent a distributed parabolic system by an unknown function.

The work presented herein has been motivated by the need to identify spatially varying parameters of building structural systems. The usefullness of some of the results obtained is illustrated through an actual example in this specific application area. Physical interpretations of the mathematical results have been provided as they are encountered.

The work presented herein has been motivated by the need to identify spatially varying parameters of building structural systems. The usefulness of some of the results obtained is illustrated through an actual example in this specific application area. Physical interpretations of the mathematical results have been provided as they are encountered.

2. System equations. Consider a system whose response $u(x, t)$ is described by the one dimensional wave equation

$$
\rho(x) \frac{\partial^{2} u}{\partial t^{2}}=\frac{\partial}{\partial x}\left[a(x) \frac{\partial u}{\partial x}\right]-b(x) u-f(x, t) .
$$

The boundary conditions are given by

$$
\begin{aligned}
& a_{0} u(0, t)-\left(1-a_{0}\right) \frac{\partial u}{\partial x}(0, t)=g_{0}(t) \text { and } \\
& b_{0} u(1, t)+\left(1-b_{0}\right) \frac{\partial u}{\partial x}(1, t)=h_{0}(t)
\end{aligned}
$$

\footnotetext{
${ }^{1}$ We thank an annonymous reviewer for having brought to our attention the work on parabolic equations.
} 
where $0 \leqslant a_{0} \leqslant 1$ and $0 \leqslant b_{0} \leqslant 1$. The initial conditions are given by

$$
\begin{aligned}
& u(x, 0)=u_{0} \text { and } \\
& \dot{u}(x, 0)=\dot{u}_{0} .
\end{aligned}
$$

We assume that

(a) $a(x), \rho(x)>0$ and belong to $C^{2}[0,1]$,

(b) $b(x)>0$ and belongs to $C^{1}[0,1]$,

(c) the constants $a_{0}$ and $b_{0}$ are known and, are not both simultaneously zero, and

(d) the forcing functions $f(x, t), g_{0}(t)$, and $h_{0}(t)$ are known.

Equation 1 may be thought of as representing the longitudinal vibrations of a rod of unit length and unit cross-sectional area whose density, $\rho(x)$, and elastic modulus, $a(x)$, vary along its length. The coefficient $b(x)$ represents an elastic restraining stiffness term. The derivative $u_{x}(x, t)$ can be interpreted as the strain in the bar and $a(x) u_{x}(x, t)$ as the stress acting at location $x$ and time $t$. As stated before, Eqs. 1-3 arise in several areas of mathematical physics. We shall use the above physical representation of the problem to interpret the mathematical results.

3. Some mathematical preliminaries. To develop the conditions for partial identification, we introduce the eigenvalue problem described by the differential equation

$$
\left(a(x) \phi_{n}^{\prime}(x)\right)^{\prime}-b(x) \phi_{n}(x)+\lambda_{n} \rho(x) \phi_{n}(x)=0
$$

and the boundary conditions

$$
\begin{aligned}
a_{0} \phi_{n}(0)-\left(1-a_{0}\right) \phi_{n}^{\prime}(0) & =0, \quad \text { and } \\
b_{0} \phi_{n}(1)+\left(1-b_{0}\right) \phi_{n}^{\prime}(1) & =0 .
\end{aligned}
$$

The eigenvectors $\left\{\phi_{n}\right\}$ form a complete orthonormal set with respect to the weighting function $\rho(x)$ [10]. The eigenvalues of Eqs. 4 and 5 can be ordered so that

$$
0<\lambda_{0}<\lambda_{1}<\lambda_{2} \cdots \lambda_{n}<\lambda_{n+1} \cdots \text {. }
$$

For the location of the measurement point, we then choose the point $x^{*}$ which belongs to the complement of the countable set $S$ given by

$$
S=\left\{x \in[0,1] \mid \phi_{n}(x)=0 \text { for some } n\right\}
$$

The solution of Eqs. 1-3 can then be expressed in terms of the nontrivial eigenfunctions $\left\{\phi_{n}\right\}$ by using the generalized eigenfunction expansion as:

$$
\begin{aligned}
u(x, t)= & \sum_{n=0}^{\infty}\left[\left(u_{0}, \phi_{n}\right) p_{n}(t)+\left(\dot{u}_{0}, \phi_{n}\right) q_{n}(t)\right] \\
& -\int_{0}^{t}\left\{\sum_{n=0}^{\infty} q_{n}(t-\tau) F_{n}(\tau) \phi_{n}(x)\right\} d \tau \\
& +\int_{0}^{t}\left\{\sum_{n=0}^{\infty} a(1)\left[\phi_{n}(1)-\phi_{n}^{\prime}(1)\right] q_{n}(t-\tau) \phi_{n}(x)\right\} h_{0}(\tau) d \tau \\
& +\int_{0}^{t}\left\{\sum_{n=0}^{\infty} a(0)\left[\phi_{n}^{\prime}(0)+\phi_{n}(0)\right] q_{n}(t-\tau) \phi_{n}(x)\right\} g_{0}(\tau) d \tau,
\end{aligned}
$$


where

$$
\begin{gathered}
p_{n}(t)=H(t) \cdot \operatorname{Cos} \sqrt{\lambda_{n}} t, \\
q_{n}(t)=\frac{H(t)}{\sqrt{\lambda_{n}}} \cdot \operatorname{Sin} \sqrt{\lambda_{n}} t, \\
H(t) \text { is the Heavyside step function, } \\
\left(f, \phi_{n}\right)=\int_{0}^{1} f(x) \rho(x) \phi_{n}(x) d x, \\
\left\langle f, \phi_{n}\right\rangle=\int_{0}^{1} f(x) \phi_{n}(x) d x, \text { and } \\
F_{n}(\tau)=\left\langle f(x, \tau), \phi_{n}(x)\right\rangle .
\end{gathered}
$$

4. Partial identification. This section deals with the identification of all the eigenvalues of the system described by Eqs. 4 and 5 through the use of (1) a suitable forcing function, (2) initial data, or (3) boundary forcing functions.

We present the following theorem on partial identification.

TheOREM 1. The eigenvalues $\left\{\lambda_{n}\right\}$ can be uniquely determined if the response $u\left(x^{*}, t\right)$, $t>0$, is observed for any of the following five loading cases.

Case 1:
(a) $u_{0}(x)=\dot{u}_{0}(x)=0$,
(b) $h_{0}(t)=g_{0}(t)=0$,
(c) $f(x, t)=f_{1}(x) f_{2}(t)$,
(d) $f_{2}(t)$ is bounded, piecewise continuous, and not null,
(e) $f_{1}(x)$ is piecewise continuous in $[0,1]$, and
(f) $\left\langle f_{1}, \phi_{n}\right\rangle \neq 0$ and is bounded for all $n$.

Case 2:

(a) $u_{0}(x)=\dot{u}_{0}(x)=0$,

(b) $f(x, t)=0$,

(c) $h_{0}(t)=0$, and

(d) $g_{0}(t)$ is bounded, piecewise continuous, and not null.

Case 3:
(a) $u_{0}(x)=\dot{u}_{0}(x)=0$,
(b) $f(x, t)=0$,
(c) $g_{0}(t)=0$, and
(d) $h_{0}(t)$ is bounded, piecewise continuous, and not null.

Case 4:
(a) $u_{0}(x)=0$,
(b) $f_{0}(t)=g_{0}(t)=0$,
(c) $f(x, t)=0$,
(d) $u_{0}$ is piecewise continuous in $(0,1)$, and
(e) $\left(\dot{u}_{0}, \phi_{n}\right) \neq 0$ for all $n$. 
Proof. Each case is separately proved as below.

Case 1: Under conditions (a)-(c), only the second term of the solution $u\left(x^{*}, t\right)$ is nonzero and is expressed as

$$
u\left(x^{*}, t\right)=-\int_{0}^{t} r_{1}\left(t-\tau ; x^{*}\right) f_{2}(\tau) d \tau, \quad t>0,
$$

where

$$
r_{1}\left(t ; x^{*}\right)=\sum_{n=0}^{\infty} \phi_{n}\left(x^{*}\right) \cdot\left\langle f_{1}, \phi_{n}\right\rangle \cdot q_{n}(t) .
$$

The eigenfunctions $\left\{\phi_{n}\right\}$ are bounded, and the eigenvalues $\left\{\lambda_{n}\right\}$ asymptomatically increase as $n^{2}[11,12]$. Furthermore, $f_{1}$, being piecewise continuous in each subinterval, causes $\left|\left\langle f_{1}, \phi_{n}\right\rangle\right| \leqslant M n^{-1}$, where $M$ is a constant, for all $n[13,14]$. Thus the series (11) is absolutely and uniformly convergent for all $t \geqslant 0$. Furthermore, the series $\Sigma(\operatorname{Sin} n t / n)$ is boundedly convergent [15], so that

$$
e^{-c x}\left|r_{1}\left(t ; x^{*}\right)\right| \text { belongs to } L_{1}(0, \infty), \text { for all } c>0 .
$$

Also by condition (d)

$$
e^{-c t}\left|f_{2}(t)\right| \text { belongs to } L_{1}(0, \infty), \text { for all } c>0 \text {. }
$$

The above two conditions thus ensure that the integral equation (10) has a unique solution, $r_{1}\left(t ; x^{*}\right)[16]$.

Let us assume that two sets of eigenvalues $\left\{\lambda_{n}\right\}$ and $\left\{\tilde{\lambda}_{n}\right\}$ exist such that identical responses $u\left(x^{*}, t\right), t>0$, are obtained to the test input function $f(x, t)$. Thus,

$$
r_{1}\left(t ; x^{*}\right)=\sum_{n=0}^{\infty} \gamma_{n}^{(1)} \frac{\operatorname{Sin} \sqrt{\lambda_{n}} t}{\sqrt{\lambda_{n}}}=\sum_{n=0}^{\infty} \tilde{\gamma}_{n}^{(1)} \frac{\operatorname{Sin} \sqrt{\lambda_{n}} t}{\sqrt{\lambda_{n}}}=\tilde{r}_{1}\left(t ; x^{*}\right),
$$

where

$$
\begin{aligned}
& \gamma_{n}^{(1)}=\phi_{n}\left(x^{*}\right)\left\langle f_{1}, \phi_{n}\right\rangle \text { and } \\
& \tilde{\gamma}_{n}^{(1)}=\tilde{\phi}_{n}\left(x^{*}\right)\left\langle f_{1}, \tilde{\phi}_{n}\right\rangle .
\end{aligned}
$$

Since the two series in (12) are both uniformly convergent, the Laplace transform of the series can be carried out by term-by-term integration. This yields

$$
\sum_{n=0}^{\infty} \frac{\gamma_{n}}{2 i \alpha_{n}}\left[\frac{1}{s-i \alpha_{n}}-\frac{1}{s+i \alpha_{n}}\right]=\sum_{n=0}^{\infty} \frac{\tilde{\gamma}_{n}}{2 i \tilde{\alpha}_{n}}\left[\frac{1}{s-i \tilde{\alpha}_{n}}-\frac{1}{s+i \tilde{\alpha}_{n}}\right]
$$

where $\alpha_{n}=\left(\lambda_{n}\right)^{1 / 4}$ and $\tilde{\alpha}_{n}=\left(\tilde{\lambda}_{n}\right)^{1 / 4}$. For the two series to yield identical results, they must have identical poles. Thus, except for a possible relabelling, $\lambda_{n}=\tilde{\lambda}_{n}$ and $\gamma_{n}=\tilde{\gamma}_{n}$, for $n \geqslant 0$. The eigenvalues are thus unique. Of course, only those terms which appear in the series representation (11) can be determined in this manner. But since $x^{*} \in S, \phi_{n}\left(x^{*}\right) \neq 0$, for all $n$. Thus the knowledge of the response $u\left(x^{*}, t\right), t>0$, of the system to the single input $f(x, t)$ which satisfies the conditions (c)-(f) guarantees unique identification of $\left\{\lambda_{n}\right\}$. 
Case 2: Under the assumptions (a)-(c), the response $u\left(x^{*}, t\right)$ reduces to

$$
u\left(x^{*}, t\right)=\int_{0}^{t} r_{2}\left(t-\tau ; x^{*}\right) g_{0}(\tau) d \tau,
$$

where

$$
r_{2}\left(t ; x^{*}\right)=\sum_{n=0}^{\infty} a(0)\left[\phi_{n}(0)+\phi_{n}^{\prime}(0)\right] \phi_{n}\left(x^{*}\right) q_{n}(t) .
$$

The function $a(x)>0$ so that $a(0)$ is not zero. Also [ $\left.\phi_{n}(0)+\phi_{n}^{\prime}(0)\right]$ cannot be zero. If it were, then Eq. 5 would imply $\phi(0)=\phi^{\prime}(0)=0$, requiring $\phi(x)$ to vanish identically. Noting that $\sqrt{\lambda_{n}} \sim n$ for large $n$, the series in Eq. 15 is seen to be uniformly convergent except at the points, $t=2 k, k \geqslant 0$. Furthermore, the series is uniformly bounded so that

$$
e^{-c t}\left|r_{2}\left(t ; x^{*}\right)\right| \text { belongs to } L_{1}(0, \infty) \text {, for all } c>0 \text {. }
$$

As before, the integral Eq. 14 thus has a unique solution, $r_{2}\left(t, x^{*}\right)$. If two different sets of eigenvalues $\left\{\lambda_{n}\right\}$ and $\left\{\tilde{\lambda}_{n}\right\}$ yield the same response, that is, if

$$
r_{2}\left(t ; x^{*}\right)=\tilde{r}_{2}\left(t ; x^{*}\right),
$$

we get

$$
\sum_{n=0}^{\infty} \gamma_{n}^{(2)} q_{n}(t)=\sum_{n=0}^{\infty} \tilde{\gamma}_{n}^{(2)} \tilde{q}_{n}(t)
$$

where

$$
\gamma_{n}^{(2)}=a(0)\left[\phi_{n}(0)+\phi_{n}^{\prime}(0)\right] \phi_{n}\left(x^{*}\right) .
$$

The series in Eq. 16 may not be uniformly convergent for all $t$, but can be taken to be equivalent in the distributional sense [17]. In fact, integrating both sides from 0 to $t$ yields

$$
\sum_{n=0}^{\infty} \gamma_{n}^{(2)} \frac{1-\operatorname{Cos} \sqrt{\lambda_{n}} t}{\lambda_{n}}=\sum_{n=0}^{\infty} \tilde{\gamma}_{n}^{(2)} \frac{1-\operatorname{Cos} \sqrt[\lambda_{n}]{ } t}{\tilde{\lambda}_{n}} .
$$

The asymptotic behavior of $\lambda_{n}$ thus ensures that each of the series in Eq. 17 is uniformly convergent and is therefore integrable. Taking Laplace transforms as before, this yields $\lambda_{n}=\tilde{\lambda}_{n}$ and $\gamma_{n}^{(2)}=\tilde{\gamma}_{n}^{(2)}$, with at most a relabelling.

Case 3: The proof is along the same lines as in Case 2.

Case 4: Using the assumptions, the solution reduces to

$$
u\left(x^{*}, t\right)=\sum_{n=0}^{\infty}\left(\dot{u}_{0}, \phi_{n}\right) q_{n}(t) \phi_{n}\left(x^{*}\right),
$$

and noting that $\dot{u}_{0}$ is piecewise continuous in $(0,1)$, we get $\left(\dot{u}_{0}, \phi_{n}\right) \leqslant M n^{-1}$, for large $n$.

Thus the series is uniformly convergent for all $\tau$. If the same response $u\left(x^{*}, t\right), t>0$, is evoked by a certain initial velocity satisfying the condition (e), by two different systems denoted by $\left\{\lambda_{n}\right\}$ and $\left\{\tilde{\lambda}_{n}\right\}$, then

$$
\sum_{n=0}^{\infty} \gamma_{n}^{(3)} q_{n}(t)=\sum_{n=0}^{\infty} \tilde{\gamma}_{n}^{(3)} q_{n}(t),
$$


where

$$
\gamma_{n}^{(3)}=\phi_{n}\left(x^{*}\right)\left(\dot{u}_{0}, \phi_{n}\right)
$$

Again, using Laplace transforms, it follows that $\lambda_{n}=\tilde{\lambda}_{n}$ and $\gamma_{n}=\tilde{\gamma}_{n}$.

4.1. Some physical interpretations of the results. The system given by Eqs. 1, 2, and 3 has been analyzed in terms of the eigenvalue problem of Eqs. 4 and 5. The eigenvalues $\left\{\lambda_{n}\right\}$ correspond to the frequencies of vibration of the rod when its ends are elastically attached. These eigenvalues can be uniquely determined by obtaining the time history of response of the system to excitations of the ends of the bar or to forcing functions applied along the bar. Response to an initial velocity input can also be used. The requirement that the point of measurement, $x^{*}$, be so chosen that $x^{*} \in S$ implies that information about a particular mode can only be obtained from data obtained at points other than the nodes of that particular mode. This then is simply the condition for the observability of the mode [4]. The unique determination of all the eigenvalues then simply requires that the response be measured at a location which is not a node for any mode of vibration of the system. If, for instance, $a_{0} \neq 1$, by Eq. $5, \phi_{n}(0) \neq 0$ and hence a suitable observation point, $x^{*}$ for obtaining the system response would be $x=0$. Similarly, the condition that $\left\langle f_{1}, \phi_{n}\right\rangle \neq 0$ for any $n$ implies that the forcing function is so chosen that each mode of the system can be excited thus making the system controllable [4].

5. Unique identification of coefficients from point measurements. We begin by considering the system defined by Eqs. 1-3 assuming that $f(x, t)=0$ and that the system starts from rest (i.e., $u_{0}=\dot{u}_{0}=0$ ). Using the transformations

$$
\nu(x, t)=\frac{\alpha(x)}{\alpha(0)} u(x, t) \text { and } y=\int_{0}^{x} \beta(x) d x,
$$

where

$$
\alpha(x)=[\rho(x) \cdot a(x)]^{1 / 4} \text { and } \beta(x)=[\rho(x) / a(x)]^{1 / 2},
$$

we get

$$
\left[\frac{\partial^{2}}{\partial y^{2}}-\frac{\partial^{2}}{\partial t^{2}}-c(y)\right] \nu(y, t)=0
$$

with the boundary conditions

$$
\begin{aligned}
& A_{0} \nu(0, t)-B_{0} \frac{\partial \nu}{\partial y}(0, t)=g_{0}(t) \text { and } \\
& A_{1} \nu(l, t)+B_{1} \frac{\partial v}{\partial y}(l, t)=h_{0}(t)
\end{aligned}
$$

and the initial condition

$$
\nu(y, 0)=\dot{\nu}(y, 0)=0,
$$


where

$$
\begin{aligned}
l & =\int_{0}^{1} \beta(x) d x, \\
A_{0} & =a_{0}+\left(1-a_{0}\right) \alpha_{y}(0) \frac{\beta(0)}{\alpha(0)}, \\
B_{0} & =\left(1-a_{0}\right) \beta(0), \\
A_{1} & =b_{0} \frac{\alpha(0)}{\alpha(l)}-\left(1-b_{0}\right) \alpha_{y}(l) \frac{\beta(l)}{\alpha(l)} \frac{\alpha(0)}{\alpha(l)}, \\
B_{1} & =\left(1-b_{0}\right) \beta(l) \frac{\alpha(0)}{\alpha(l)}, \text { and } \\
c(y) & =\left[\frac{b(y)}{\rho(y)}+\frac{1}{\alpha(y)} \frac{d^{2} \alpha(y)}{d y^{2}}\right] .
\end{aligned}
$$

We note by Eq. 20 that

$$
\nu(y=0, t)=\nu(x=0, t)=u(0, t) .
$$

Consider now the eigenfunction expansion

$$
\nu(y, t)=\sum \tilde{\nu}_{n}(t) \psi_{n}(y)
$$

where the functions $\psi_{n}$ satisfy the equations

$$
\psi_{n}^{\prime \prime}+\left(\lambda_{n}-c(y)\right) \psi_{n}=0
$$

and

$$
\begin{aligned}
& A_{0} \psi(0)-B_{0} \psi^{\prime}(0)=0, \\
& A_{1} \psi(l)+B_{1} \psi^{\prime}(l)=0 .
\end{aligned}
$$

The functions $\left\{\psi_{n}\right\}$ are orthogonal and complete so that

$$
\tilde{\nu}_{n}(t)=\frac{1}{\eta_{n}} \int_{0}^{l} \nu(y, t) \psi_{n}(y) d y,
$$

where

$$
\eta_{n}=\int_{0}^{l} \psi_{n}^{2}(y) d y .
$$

We assume that $a_{0}$ and $b_{0}$ are not unity, i.e., $B_{0}$ and $B_{1}$ are not zero (the case when either $a_{0}$ or $b_{0}$ (or both) equal unity will be dealt with later). Using the expansion given in Eq. 28, the solution to Eqs. 22, 23, and 24 can now be written as:

$$
\begin{aligned}
\nu(y, t)= & \int_{0}^{t}\left\{\sum_{n=0}^{\infty} \frac{\psi_{n}(y) \psi_{n}(0)}{\eta_{n} B_{0}} q_{n}(t-\tau)\right\} g_{0}(\tau) d \tau \\
& \left.+\int_{0}^{t}\left\{\sum_{n=0}^{\infty} \frac{\psi_{n}(y) \psi_{n}(l)}{\eta_{n} B_{1}} q_{n}(t-\tau)\right\} h_{0}(\tau) d \tau\right\} .
\end{aligned}
$$

6. Identification of the coefficients $a(x)$. We now present some theorems which provide a sufficient condition for the unique identification of $a(x)$ (given the functions $\rho(x)$ and $b(x))$ from observations made at a single location. 
THEOREM 2 . The coefficient $a(x)$ in Eq. 1 can be uniquely identified from measurements $u(0, t), t>0$, if the following conditions are satisfied.

(1) $\rho(x), b(x)$ are known, $x \in[0,1]$,

(2) $a_{0} \neq 1$, i.e., $B_{0} \neq 0$,

(3) $a(0), a_{x}(0), a(1)$, and $a_{x}(1)$ are known,

(4) $c(y)>0, y \in(0, l)$

(5) $f(x, t)=h_{0}(t)=0$, for all $t$, and

(6) $g_{0}(t)$ is not a null function, is bounded, and is known for $t \geqslant 0$.

Proof. Since $B_{0} \neq 0$, the eigenfunctions $\psi_{n}$ can be normalized so that $\psi_{n}(0)=1$.

Also,

$$
\alpha_{y}(0)=\frac{\alpha_{x}(0)}{\beta(0)}=f\left(a_{x}(0), a(0)\right)
$$

thus condition (3) implies that the constants $A_{0}, B_{0}, A_{1}$, and $B_{1}$ are all known. Furthermore, knowledge of $u(x=0, t), t>0$, implies knowledge of $\nu(y=0, t), t>0$. Using Eq. 30 ,

$$
\nu(y, t)=\int_{0}^{t}\left\{\sum_{n=0}^{\infty} \frac{\psi_{n}(y)}{\eta_{n} B_{0}} q_{n}(t-\tau)\right\} g_{0}(\tau) d \tau
$$

Thus

$$
\nu(0, t)=\int_{0}^{t}\left\{\sum_{n=0}^{\infty} \frac{q_{n}(t-\tau)}{\eta_{n} B_{0}}\right\} g_{0}(\tau) d \tau .
$$

As before, the eigenvalues $\left\{\lambda_{n}\right\}$ are all real and positive, and increase asymptotically as $n^{2}$. Thus, the integral Eq. 34 whose left hand side is known (Eq. 27), has a unique solution [16] given by

$$
r(t)=\sum_{n=0}^{\infty} \frac{q_{n}(t)}{\eta_{n} B_{0}} .
$$

The series $r(t)$, as before, is not uniformly convergent, but can be interpreted in a distributional sense. If two sets of eigenvalues $\left\{\lambda_{n}\right\}$ and $\left\{\tilde{\lambda}_{n}\right\}$ exist so that they yield the same function $r(t)$, then by taking Laplace Transforms it can again be shown that

$$
\lambda_{n}=\tilde{\lambda}_{n} \text { and } \eta_{n}=\tilde{\eta}_{n} .
$$

Thus the eigenvalues $\left\{\lambda_{n}\right\}$ and the set $\left\{\eta_{n}\right\}$ are uniquely determinable. Use of the spectral function $S(\lambda)$, defined by

$$
S(\lambda)=\sum_{n=0}^{\infty} \eta_{n}^{-1}, \text { for } \lambda_{n} \leqslant \lambda
$$

then guarantees the unique determination of the function $c(y)$ [18]. Once $c(y)$ is known, the differential Eq. 26 together with condition (3) yields $a(x)$ uniquely.

It is seen that the proof of the theorem critically depends upon the parameters $a_{0}$ and $b_{0}$. Clearly, if $a_{0} \neq 1$ and $b_{0}=1$, unique identification of the coefficient $a(x)$ can be 
guaranteed by the previous theorem without an explicit knowledge of $a(1)$ and $a_{x}(1)$ since these terms drop out from the expression for $A_{1}$ and from Eq. 30 for that case.

We note that the requirement $c(y)>0$ for $y \in(0, l)$, though it appears to be a limitation, is often satisfied by stable oscillating systems. On the other hand, $b_{0} \neq 1$ implies $B_{1} \neq 0$, and we have the following theorem.

Theorem 3. The coefficient $a(x)$ in Eq. (1) can be uniquely identified rom measurements $u(1, t), t>0$, if the following conditions are satisfied.

(1) $\rho(x), b(x)$ are known, $x \in[0,1]$,

(2) $b_{0} \neq 1$, i.e., $B_{1} \neq 0$,

(3) $a(0), a_{x}(0), a(1)$, and $a_{x}(1)$ are known,

(4) $c(y)>0$, for $y \in(0, l)$,

(5) $f(x, t)=g_{0}(t)=0$, and

(6) $h_{0}(t)$ is not a null function, is bounded, and is known for $t \geqslant 0$.

Proof. We now use the substitution

$$
\nu(x, t)=\frac{\alpha(x)}{\alpha(1)} u(x, t)
$$

instead of that used in Eq. 20. Relations given in Eqs. 22 and 23 are valid, except that the constants $A_{0}, B_{0}, A_{1}$, and $B_{1}$ are replaced by $A_{0}^{\prime}, B_{0}^{\prime}, A_{1}^{\prime}$, and $B_{1}^{\prime}$, respectively, where each of the primed quantities equal $\alpha(l) / \alpha(0)$ times the corresponding unprimed quantity. Using Eq. 32, we get

$$
\nu(x=1, t)=\int_{0}^{t}\left\{\sum_{n=0}^{\infty} \frac{q_{n}(t-\tau)}{\eta_{n} B_{1}}\right\} h_{0}(\tau) d \tau,
$$

where the set $\left\{\psi_{n}\right\}$ is normalized so that $\psi_{n}(l)=1$ for all $n$, since $B_{1} \neq 0$.

Noting that $\nu(l, t)=u(1, t)$, the remainder of the proof follows the same lines as the previous theorem. The function $c(y)$ is uniquely determined, and $a(x)$ is obtained by integrating the differential Eq. 26 using the known initial conditions $a(1)$ and $a_{x}(1)$. Once again, if $a_{0}=1$ and $b_{0} \neq 1$, knowledge of neither $a_{x}(0)$ nor $a(0)$ is required for the identification of the coefficient $a(x)$.

We now consider the case in which either $a_{0}$ or $b_{0}$ (or both) equal unity, implying thereby, that $B_{0}$ or $B_{1}$ (or both) equal to zero. Unique identification of the coefficient $a(x)$ can be again achieved by a strain measurement made at one point in the spatial domain.

THEOREM 4. The coefficient $a(x)$ in Eq. 1 can be uniquely identified from measurements $u_{x}(0, t), t>0$, if the following conditions are met.

(1) $\rho(x), b(x)$ are known for $x \in[0,1]$,

(2) $a_{0}=1$, i.e., $B_{0}=0$,

(3) $a(0), a_{x}(0), a(1)$ and $a_{x}(1)$ are known,

(4) $c(y)>0$ for $y \in(0, l)$

(5) $f(x, t)=h_{0}(t)=0$, and

(6) $g_{0}(t)$ is not a null function, is bounded, and is known for $t \geqslant 0$. 
Proof. Using Eqs. 20-27, and the eigenfunction expansion given in Eq. 28, the solution for $\nu_{y}(x, t)$ can be written as

$$
\nu_{y}(0, t)=\int_{0}^{t}\left\{\sum_{n=0}^{\infty} \frac{1}{\eta_{n}} q_{n}(t-\tau)\right\} g_{0}(\tau) d \tau,
$$

where we have normalized the set $\left\{\psi_{n}\right\}$ so that $\psi_{n}^{\prime}(0)=1$ for all $n$. But

$$
\frac{1}{\alpha(0)} \frac{\partial u}{\partial x}(x, t)=\frac{-\nu(x, t)}{\alpha^{2}(x)} \frac{d \alpha}{d x}+\frac{\beta(x)}{\alpha(x)} \frac{\partial \nu}{\partial y},
$$

so that

$$
\frac{\partial u}{\partial x}(0, t)=\frac{-g_{0}(t)}{\alpha(0)} \alpha_{x}(0)+\beta(0) \nu_{y}(0, t) .
$$

Thus the knowledge of $\partial u(0, t) / \partial x$ in addition to the conditions of the theorem leads to a knowledge of $\nu_{y}(0, t)$ for all time.

Once again, the coefficients $A_{0}, A_{1}$ and $B_{1}$ are all known, and Eq. 39 has a unique solution which further enables a unique determination of $\left\{\lambda_{n}\right\}$ and $\left\{\eta_{n}\right\}$ and thus a unique determination of $a(x)$.

If the functions $a(x)$ and $b(x)$ are known, then unique identification of $\rho(x)$ can be guaranteed by replacing $a(\cdot)$ by $\rho(\cdot)$ in Thms. 2, 3, and 4. The proofs follow directly from those presented in the theorems.

In order to interpret the results, we shall think in terms of the vibrating rod under various types of boundary conditions.

Consider first the system wherein $b_{0}=0$ (so that $B_{1} \neq 0$ ) and $a_{0}=1$. For $g_{0}(t)=0=$ $f(x, t)$ and $h_{0}(t) \neq 0$, the problem is then a mathematical description of a rod fixed at the end $x=0$ and subjected to a stress time history $\sigma(1, t)=h_{0}(t) a(1)$ at the end $x=1$. Theorem 3 then states that if the response $u(1, t)$ is measured for the applied stress history at $x=1$, then unique identification of the $a(x)$ is guaranteed if $a(1)$ and $a_{x}(1)$ are known a priori. Note that the term involving $a_{x}(0)$ in Eq. 25 drops out making its knowledge unessential as far as unique identification of $a(x)$ is concerned.

Figure 1 shows the quantities which when known for all time yield unique identification of $a(x)$. The same set of boundary conditions can also be used with the results of Thm. 4

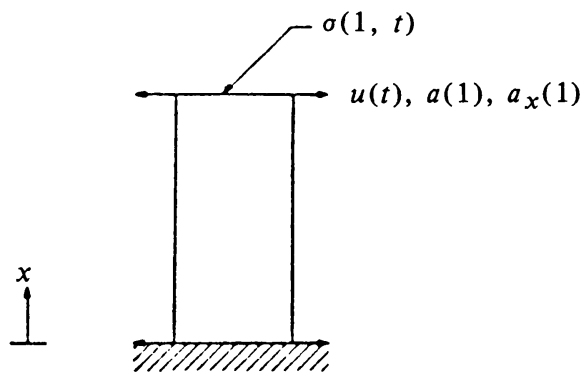

FIG. 1. Boundary conditions for a vibrating rod fixed at one end that allow a unique identification of $a(x)$. 


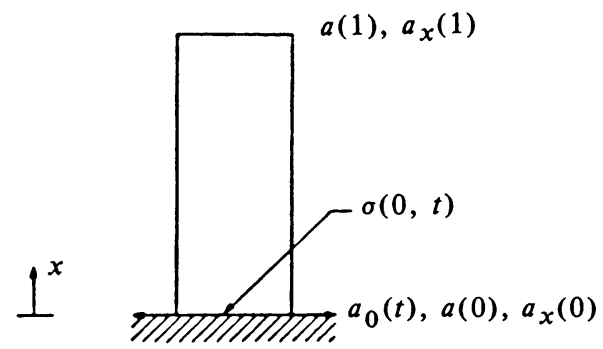

FIG. 2. Boundary conditions for a vibrating rod fixed at one end that allow a unique identification of $a(x)$.

which guarantees that if the base $(x=0)$ is vibrated with a motion $g_{0}(t)$ and the stress $\sigma(0, t)$ is measured for all $t>0$, a fore knowledge of $a(1), a_{x}(1), a(0)$ and $a_{x}(0)$ will guarantee unique identification of $a(x)$. We note that the last two quantities, $a(0)$ and $a_{x}(0)$, are required because of Eq. 40. In many practical applications it may be assumed that $a_{x}(0)=a_{x}(1)=0$. Then by virtue of Eq. 25 , only $a(0)$ requires to be known in addition to the stress $\sigma(0, t)$ for $t \geqslant 0$, to obtain unique identification.

Figure 2 shows the data required to uniquely identify the coefficient $a(x)$. We observe the reciprocity in the requirement for unique identification: the measurement of displacement at the free end caused by an applied stress history at that end is equivalent to the measurement of the stress history at the fixed end induced by base displacements at that end, so far as guaranteeing unique identification is concerned. However, differences in the requirements in the knowledge of $a(x)$ and its derivative at the end point $x=0$ should also be noticed.

We note in passing that Thm. 4 is also valid for the case of the fixed-fixed boundary conditions when both $a_{0}$ and $b_{0}$ equal unity.

\section{Identification of the coefficient $b(x)$.}

THEOREM 5. The coefficient $b(x)$ in Eq. (1) is uniquely identified from the measurement of response, $u(1, t), t>0$, induced by the forcing function $g(t)$ if the following conditions are met.

(a) $\rho(x), a(x)$ are known in $[0,1]$,

(b) $a_{0} \neq 1$, i.e., $B_{0} \neq 0$,

(c) $c(y)>0$ for $y \in(0, l)$,

(d) $f(x, t)=h_{0}(t)=0$ for all $t$, and

(e) $g_{0}(t)$ is not a null function, is bounded, and is known for $t \geqslant 0$.

THEOREM 6. The coefficient $b(x)$ in equation (1) can be uniquely identified from response measurements $u_{x}(0, t), t>0$, to the forcing function $g_{0}(t)$, if

(1) $\rho(x), a(x)$ are known $\rho(x), a(x)$ are known in $[0,1]$,

(2) $a_{0}=1$, i.e., $B_{0}=0$,

(3) $c(y)>0, y \in(0, l)$, 
(4) $f(x, t)=h_{0}(t)=0$ for all time $t$, and

(5) $g_{0}(t)$ is not a null function, is bounded, and is known for $t \geqslant 0$.

Proof. The proofs of Thms. 5 and 6 are along the same lines as those of Thms. 2 and 4 .

We note that no a priori knowledge of $b(x)$ or its derivatives at the end points $x=0$ or $x=1$ is required for unique identification.

8. Discussion and applications. This paper looks at the problem of identification of vibrating systems that are described by second order hyperbolic differential equations. Conditions for identification of the spatially distributed parameters are obtained from measurements made at one point of the spatial domain.

Conditions for unique identification of all the eigenvalues of the system are obtained. It is shown that under suitable restrictions on the nature of the various functions concerned (which are in practice almost always met with in physical systems), unique identification of all the eigenvalues can be obtained by measurement of the response to either a forcing function of the form $f(x, t)=f_{1}(x) f_{2}(t)$ or to end excitations $g_{0}(t)$ and $h_{0}(t)$. The response measurement needs to be obtained (for all time) at a location which is not a node of any mode of vibration of the system.

From the structure of the related eigenvalue problem (Eqs. 28 and 29), it is clear that, in general, more than one out of three spatially varying coefficients cannot be uniquely identified through point measurements. However, knowledge of any two can yield unique identification of the third when suitable a priori information about the unknown coefficient at the two end points of the domain is available. The conditions for identifying $\rho(x)$, given $a(x)$ and $b(x)$, are analogous to those for identifying $a(x)$ given $\rho(x)$ and $b(x))$. The measurement location for identifying the coefficients in each case is one end of the spatial domain.

The application area which has motivated this study is the identification of building structural systems from records obtained during strong earthquake ground shaking. Figures 1 and 2 were chosen as specific examples of the commonly used shear-beam type models of tall building structures. Whereas the classical paper of Levinson [19] provides unique identification of the coefficients $c(y)$ in Eq. 28, if all the eigenvalues $\left\{\lambda_{n}\right\}$ can be obtained for two different sets of boundary conditions 29, actual tests on large structural systems which would yield such data are often difficult to perform. For instance, a tall building structure whose horizontal vibrations are often modelled by a cantilever shear beam $(a(x)$ is then related to the stiffness in shear) cannot be tested easily under two different boundary conditions. The stiffness distribution, $a(x)$ of such a structure (for which $a_{0}=1$ and $b_{0}=0$ ) can thus be uniquely identified, if the stress induced at the base of the structure by a ground shaking $g_{0}(t)$ can be determined, provided the stiffness $a(0)$ (assuming $a_{x}(0)=a_{x}(1)=0$ ) is known a priori. This result is a natural extension of the results on discrete structural models wherein unique identification of the interstory stiffness is obtained through measurement of the displacement response of the first story level and that of the base motion [5].

The results presented herein will also find important applications in the area of soil-structure interaction. For instance, Thms. 5 and 6 could apply to the estimation of soil 
stiffness parameters along the length of piles which are driven into the ground. It is hoped that some of these results may be useful in other areas of mathematical physics and engineering science as well.

\section{REFERENCES}

[1] M. Balas, Modal control of certain flexible dynamic systems, SIAM J. Control, 16, pp. 450-462 (1978)

[2] F. E. Udwadia and P. C. Shah, Identification of building structural systems from records obtained during strong ground motion, J. Engg. and Industry, ASME Transactions, 98, 1347-1362 (1976)

[3] F. E. Udwadia and D. K. Sharma, Some uniqueness results related to building structural identification, SIAM J. Applied Math., 34, 104-118 (1978)

[4] F. E. Udwadia, Controllability, observability and identification of classical linear dynamic systems, Solid Mechanics Archives, 6, (1981)

[5] F. E. Udwadia, D. K. Sharma and P. C. Shah, Uniqueness of damping and stiffness distributions in the identification of soil and structural systems, J. Applied Mech., 45, 181-187 (1978)

[6] D. Luenberger, Introduction to Linear and Nonlinear Programming, Addison Wesley, 1965

[7] M. M. Sondhi and B. Gopinath, Determination of vocal-tract shape from impulse response at the lips, J. Acoustical Soc. Amer., 49, 1867-1873 (1971)

[8] B. Gopinath and M. M. Sondhi, Inversion of the telegraph equation and the synthesis of nonuniform lines, Proc. IEEE, 59, 383-392 (1971)

[9] R. Burridge, The Gelfand-Levitan, the Marchensko, and the Gopinath-Sondhi integral equations of inverse scattering theory, regarded in the context of inverse-response problems, Wave Motion, 2, 305-323 (1980)

[10] E. L. Ince, Ordinary Differential Equations, Dover, pp. 235-240

[11] E. L. Ince, Ordinary Differential Equations, Dover

[12] Hilbert and Courant, Methods of Mat. Physics, Vol. I, Interscience Publishers, 1966

[13] E. L. Ince, Ordinary Differential Equations, Dover, pp. 275-278

[14] I. Stakgold, Green's Functions and Boundary Value Problems, Wiley, 1979

[15] E. C. Titchmarsh, The Theory of Functions, Oxford Press, 1979

[16] E. C. Titchmarsh, Theory of the Fourier Integral, Oxford Press, 1935

[17] I. Stakgold, Green's Functions and Boundary Value Problems, Wiley, 1979

[18] I. M. Gelfand and B. M. Levitan, On the determination of a differential equation from its spectral function, Izvestiya Akad. Nauk SSR, 15, 309-360 (1951)

[19] N. Levinson, The inverse Sturm-Liouville problem, Mat. Tidsskr, B, 25-30 (1949)

[20] A. Bamberger, G. Chavent and P. Lailly, Etude mathematique et numerique d'un probleme inverse pour l'equation des ondes a' une dimension, Report LABORIA, No. 226, IRIA

[21] S. Kitamura and S. Nakagiri, Identifiability of spatially-varying and constant parameters in distributed systems of parabolic type, SIAM J. Control and Optimization, 15, 785-802 (1977)

[22] A. Pierce, Unique identification of eigenvalues and coefficients in a parabolic problem, SIAM J. Control and Optimization, 17 494-499 (1979)

[23] T. Kobayashi, Determination of unknown functions for a class of distributed parameter systems, SIAM J. Control and Optimization, 17 469-476 (1979) 